\title{
Kloosterhuis, Jürgen, Neugebauer, Wolfgang, Krise, Reformen und Finanzen. Preußen vor und nach der Katastrophe von 1806
}

Guillaume Garner

\section{OpenEdition}

\section{Journals}

Édition électronique

URL : http://journals.openedition.org/ifha/2121

DOI : 10.4000/ifha.2121

ISSN : 2198-8943

Éditeur

IFRA - Institut franco-allemand (sciences historiques et sociales)

Référence électronique

Guillaume Garner, « Kloosterhuis, Jürgen, Neugebauer, Wolfgang, Krise, Reformen und Finanzen. Preußen vor und nach der Katastrophe von 1806 », Revue de I'IFHA [En ligne], Date de recension, mis en ligne le 01 janvier 2010, consulté le 22 septembre 2020. URL : http://journals.openedition.org/ifha/ 2121 ; DOI : https://doi.org/10.4000/ifha.2121

Ce document a été généré automatiquement le 22 septembre 2020.

(CIFHA 


\title{
Kloosterhuis, Jürgen, Neugebauer, Wolfgang, Krise, Reformen und Finanzen. Preußen vor und nach der Katastrophe von 1806
}

\author{
Guillaume Garner
}

1 Ce volume collectif vise à étudier l'histoire des finances prussiennes à l'époque de la Sattelzeit, qui constitue un domaine paradoxalement peu connu, ou plutôt mal connu, dans la mesure où le regard s'est très souvent porté sur le niveau de l'administration centrale, au détriment des échelles locale et régionale. Ce faisant, l'objectif affiché par J.K. et W.N. dans leurs deux textes introductifs est d'examiner les implications de la question financière, non seulement dans le domaine de la politique étrangère - ce sujet n'est l'objet que d'une seule contribution, signée P. Baumgart - mais également dans le domaine politique et notamment par rapport à la question constitutionnelle (Verfassungsfrage).

2 Réunissant des spécialistes reconnus, ce volume, dont le contenu très riche et dense rend un résumé par trop réducteur, remplit largement ses objectifs grâce à un triple élargissement. Le premier élargissement est thématique, vers les questions politiques et sociales. W. Neugebauer démontre ainsi de manière convaincante que la position des diètes régionales ne peut unilatéralement être qualifiée de conservatrice et d'antiréformatrice : au contraire, la crise financière après 1806 a encouragé la modernisation des programmes politiques de ces diètes en promouvant l'idée de participation politique à travers une évolution constitutionnelle (certes modérée). L'opposition entre réformateurs (au premier rang desquels Alexander von Humboldt) et opposition conservatrice est également au centre de l'étude de R. Zilch sur la question du financement des réformes du système éducatif, tandis que $M$. Winter retrace les conflits d'intérêts au centre du débat sur la répartition des charges de guerre dans la ville de Berlin en 1809. 
3 Le deuxième élargissement est chronologique : une grande partie des contributions s'attache à replacer les débats et les réformes dans le domaine financier dans une moyenne durée, allant du milieu du XVIIe siècle au milieu du XIXe siècle. Le balancement entre rupture et continuité est ainsi au centre des contributions de H.-C. Kraus sur le traitement de la question financière dans le caméralisme et l'économie politique, tandis que K.H. Kaufhold souligne combien les réformes de la statistique administrative impulsées par Johann Gottfried Hoffmann après 1806 ont été une réussite car l'État prussien disposait enfin des moyens de parvenir à la saisie des richesses du pays que prônaient les caméralistes depuis le début du XVIIIe siècle.

Le troisième élargissement est géographique : loin d'être centré sur la seule Prusse, ce recueil consacre une partie à des études de cas se référant à l'espace germanophone : le rapport de la noblesse et des diètes westphaliennes à la question fiscale est examiné par H.-J. Behr, tandis que W. Buchholz souligne à quel point la préservation des intérêts financiers de la noblesse foncière en Poméranie occidentale a bloqué toute amélioration de la condition paysanne pendant le XIXe siècle. Le point de vue des finances étatiques est envisagé par les contributions de I. Schnelling-Reinicke sur les États napoléoniens ; H.-H. Brandt montre que la banqueroute autrichienne de 1811 a été suivie de réformes dont la portée a été amoindrie par le manque d'intégration politique et économique du territoire de la monarchie, et H.-P. Ullmann souligne que les États de l'Allemagne du Sud ont certes mené du début du XIXe siècle aux années 1840 une politique de réduction de la dette publique, mais que les effets de celle-ci ont été annulés par la prise en charge de nouvelles missions, dans le domaine de l'éducation et des infrastructures (ferroviaires).

5 J.K. et W.N. ne prétendaient certes pas épuiser un si vaste sujet, et soulignent euxmêmes la nécessité de mener des études de cas à l'échelle locale et régionale ; il n'en demeure pas moins qu'ils proposent un panorama bien informé et ouvert aux multiples dimensions des thématiques étudiées.

Guillaume Garner (IFHA) 